\title{
Utilizing data sampling techniques on algorithmic fairness for customer churn prediction with data imbalance problems
}

\section{[version 1; peer review: 2 approved with reservations]}

\author{
Maw Maw, Su-Cheng Haw (D), Chin-Kuan Ho \\ Faculty of Computing and Informatics, Multimedia University, Cyberjaya, Selangor, 63100, Malaysia
}

V1 First published: $30 \operatorname{Sep} 2021,10: 988$

https://doi.org/10.12688/f1000research.72929.1

Latest published: 27 Jun 2022, 10:988

https://doi.org/10.12688/f1000research.72929.2

\section{Abstract}

Background: Customer churn prediction (CCP) refers to detecting which customers are likely to cancel the services provided by a service provider, for example, internet services. The class imbalance problem (CIP) in machine learning occurs when there is a huge difference in the samples of positive class compared to the negative class. It is one of the major obstacles in CCP as it deteriorates performance in the classification process. Utilizing data sampling techniques (DSTs) helps to resolve the CIP to some extent.

Methods: In this paper, we review the effect of using DSTs on algorithmic fairness, i.e., to investigate whether the results pose any discrimination between male and female groups and compare the results before and after using DSTs. Three real-world datasets with unequal balancing rates were prepared and four ubiquitous DSTs were applied to them. Six popular classification techniques were utilized in the classification process. Both classifier's performance and algorithmic fairness are evaluated with notable metrics.

Results: The results indicated that Random Forest classifier outperforms other classifiers in all three datasets and, using SMOTE and ADASYN techniques cause more discrimination in the female group. The rate of unintentional discrimination seems to be higher in the original data of extremely unbalanced datasets under the following classifiers: Logistics Regression, LightGBM, and XGBoost. Conclusions: Algorithmic fairness has become a broadly studied area in recent years, yet there is a very little systematic study on the effect of using DSTs on algorithmic fairness. This study presents important findings to further the use of algorithmic fairness in CCP research.

Keywords

Customer churn prediction, Data sampling techniques, Algorithmic fairness, Class imbalance problem

\section{Open Peer Review \\ Approval Status ? ? \\ 12 \\ 23 \\ version 2 \\ (revision) \\ 27 Jun 2022

$\begin{array}{lcc}\text { version } 1 & ? & ? \\ 30 \text { Sep 2021 } & \text { view } & \text { view }\end{array}$

1. Chu Kiong Loo ID, University of Malaya,

Kuala Lumpur, Malaysia

2. Prabu P ID, CHRIST University, Bengaluru, India

3. Kok-Chin Khor ID, Universiti Tunku Abdul Rahman, Selangor, Malaysia

Any reports and responses or comments on the article can be found at the end of the article. 


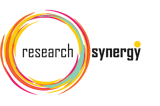

sing

This article is included in the Research Synergy

Foundation gateway.

Corresponding author: Su-Cheng Haw (sucheng@mmu.edu.my)

Author roles: Maw M: Data Curation, Formal Analysis, Investigation, Methodology, Writing - Original Draft Preparation; Haw SC: Funding Acquisition, Project Administration, Supervision, Writing - Review \& Editing; Ho CK: Project Administration, Writing - Review \& Editing

Competing interests: No competing interests were disclosed.

Grant information: This work is supported by the funding of TM Research \& Development from Telekom Malaysia, Malaysia (Ref: MMUE/160013).

The funders had no role in study design, data collection and analysis, decision to publish, or preparation of the manuscript.

Copyright: @ 2021 Maw M et al. This is an open access article distributed under the terms of the Creative Commons Attribution License, which permits unrestricted use, distribution, and reproduction in any medium, provided the original work is properly cited.

How to cite this article: Maw M, Haw SC and Ho CK. Utilizing data sampling techniques on algorithmic fairness for customer churn prediction with data imbalance problems [version 1; peer review: 2 approved with reservations] F1000Research 2021, 10:988 https://doi.org/10.12688/f1000research.72929.1

First published: 30 Sep 2021, 10:988 https://doi.org/10.12688/f1000research.72929.1 


\section{Introduction}

Customer churn, the phenomenon in which customers are shifting to rival companies due to dissatisfaction with the existing services or to other inevitable reasons, ${ }^{1}$ is one of the common issues usually encountered in every customeroriented sector, including telecommunication. Customer churn prediction (CCP) is a supervised binary classification procedure that detects the potential churners before they are churned. Since there are no standardized principles for collecting data for CCP tasks, data distribution between classes will be varied from one data set to another. Therefore, one class might have extremely underrepresented compared to another class. In CCP, the target class is those being churned or not. To be exact, churn is always a minority class when the non-churn class usually comes in large numbers. Therefore, churn is used to consider a rare object ${ }^{2}$ in service-based domains including telecom. Thus, telecom datasets always suffer from a class imbalance problem (CIP) and lead to a situation in which minority instances remain unlearned.

Advanced machine learning techniques can be applied to predict potential churners. Let us consider a dataset with 10,000 data instances with $10 \%$ of churn samples i.e., 1000 churners and 9,000 non-churners. Even if a carefully built model could predict $90 \%$ correctly on the minority class, it means 100 customers are misclassified to the wrong class. Suppose 60 churners are misclassified as non-churners, i.e., false negatives, the company will lose a huge amount of revenue since recruiting new customers is more expensive than keeping the existing ones. ${ }^{3}$ Thus, the ultimate goal in the telecom sector is to increase profit by decreasing customer churn. Hence, CIP is a block when trying to achieve the major goal of CCP, since it degrades classification accuracy. Algorithmic fairness has become a very active research topic since ProPublica observed that the algorithms could yield discriminative outcomes, which impacted a minority group in real life. ${ }^{4}$

Algorithmic fairness is monitored in line with the protected features or sensitive variables in the dataset. Sensitive data could generally be, but not limited to gender, race, age group or religion. Algorithmic fairness is achieved if the decisions generated by a model do not favor more or less any individual or a group. ${ }^{5}$ The lesser the bias in the training data, the bigger the chance of achieving algorithmic fairness. However, it is almost not possible to train a zero-bias model since the historical data could have contained bias for many reasons. ${ }^{6}$ The common reasons for bias in the training data involve the compounding of initial bias over time, using proxy variables, and unbalancing of sample size between minority and majority group. ${ }^{7}$

In the CCP process, customers' behavior is analyzed within specific time windows, for example within one month. ${ }^{8}$ Once the prediction is done, the outcomes are reused as training data for the next prediction. Therefore, there are high chances to have repeated bias in the historical data without even noticing. One solution for CIP is to apply data sampling techniques (DSTs) to the training data. Since the major function of DSTs is to increase or decrease the sample instances to balance between majority and minority classes, there are changes in the number of samples in the different groups in the dataset. The main goal of this study is to explore and identify the impact of using DSTs on training data on algorithmic fairness in the CCP process. To the best of our knowledge, there is very little research concerning algorithmic fairness in the CCP process. We believe the findings of this study would provide valuable insights to future CCP research.

\section{Methods}

Ethical Approval Number: EA1742021

Ethical Approval Body: Research Ethics Committee 2021, Multimedia University

In this study, the original data set is prepared to make three versions of unbalanced datasets, with rates of 5\%, $15 \%$ and $30 \%$. Each version is applied with four DSTs and compared the results with the unsampled original dataset to evaluate the classification performance and impacts on algorithmic fairness. The step-by-step methods to conduct the study are presented in Figure 1.

\section{Datasets}

A real-world telecom dataset was provided by one of Malaysia's leading telecom companies (see Underlying data for details on access to this dataset). The original dataset contains 1,265,535 customer records, which were collected from January 2011 to December 2011. Since the original data set is huge in volume, we randomly selected 100,000 records and utilized them for this study. We included demographics, call information, network usage, billing information, and customer satisfactory data in our dataset since they are considered as influential factors in the CCP process. ${ }^{9,10}$ A total of 22 features were extracted after careful aggregation, i.e., new features were created based on the original data and some unnecessary features were deleted from it, and features are listed in Table 1. 


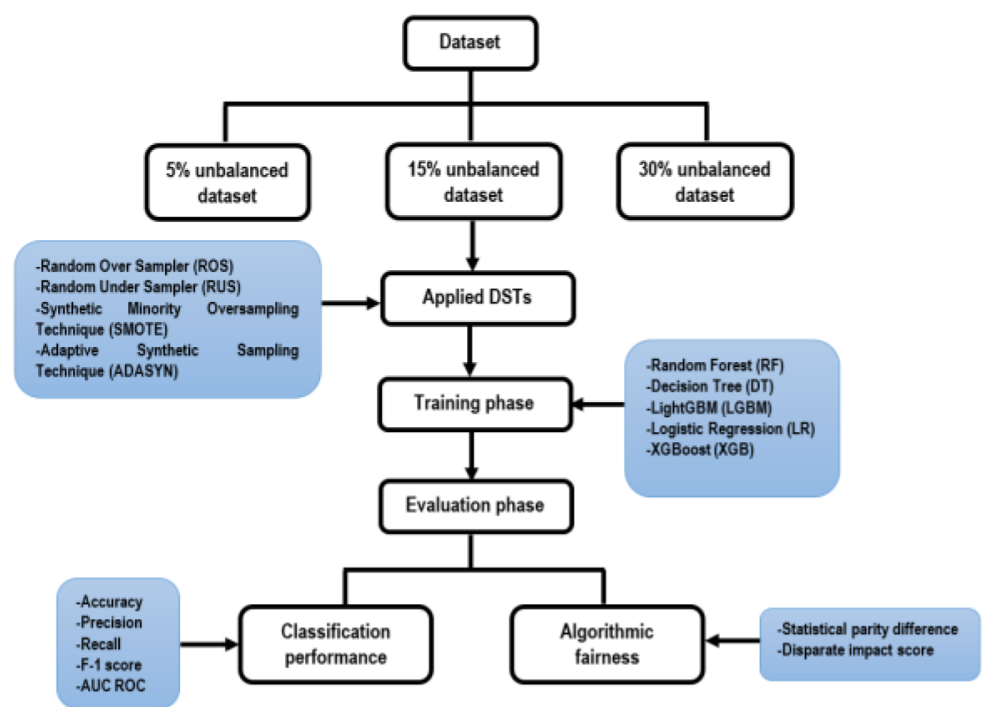

Figure 1. Procedures of the study.

\section{Table 1. Features used in the real-world dataset.}

\begin{tabular}{|c|c|c|}
\hline No. & Name of the features & Description \\
\hline 1 & Customer ID & Customer ID \\
\hline 2 & Age & Age of customer \\
\hline 3 & Is senior & Is the customer over 60 or not \\
\hline 4 & Gender & Gender of customer \\
\hline 5 & Is local & Is the customer a Malaysian or an international? \\
\hline 6 & Race & Is the customer Malay or Indian or Chinese or Other? \\
\hline 7 & Technical-problem-count & Total technical complaints and general complaints made by a customer \\
\hline 8 & Complain-count & Total general complaints made by a customer \\
\hline 9 & Avrg download & Average download rate \\
\hline 10 & Avrg upload & Average upload rate \\
\hline 11 & T-Location & The location where the customer registered for the service \\
\hline 12 & HSBB area & Is the customer in the area where a high-speed connection is required or not \\
\hline 13 & Speed & Broadband speed customer has registered for \\
\hline 14 & Price start & The value of package customer has bought \\
\hline 15 & Contract period & The contract period of the customer \\
\hline 16 & Median- outstanding & Average overdue fees \\
\hline 17 & Avrg local amt & Average amount spends for calling locally \\
\hline 18 & Avrg std amt & Average amount spends for subscriber trunk dialing \\
\hline 19 & Avrg idd amt & Average amount spends on international calls \\
\hline 20 & Avrg voice usage & Average amount spends on voice calls \\
\hline 21 & Avrg dialup amt & Average amount spends on dialup service \\
\hline 22 & Churn & Whether the customer is churned or not \\
\hline
\end{tabular}

The final dataset was prepared with three different rates of unbalancing: 5\%, 15\%, and 30\%. We created a Python script (see Extended data) which used the Pandas tool of Scikit-learn machine learning library to prepare three versions of datasets. We set up these specific rates because we wanted to experiment with extremely unbalanced cases up to intermediate levels. 
Data preprocessing

In the data preprocessing stage, we excluded any null values. Since we found only a few outliers in the selected dataset, we manually removed them without using any specific procedure. We applied four DSTs to the data: Random Over Sampler (ROS), Random Under Sampler (RUS), ${ }^{11}$ Synthetic Minority Oversampling Technique (SMOTE), ${ }^{12}$ and Adaptive Synthetic Oversampling Technique (ADASYN). ${ }^{13}$ The selection of DSTs was based on their popularity and to know the impact of each of them on the algorithmic fairness in the $\mathrm{CCP}$ process.

\section{Classification of data}

We applied six popular classifiers: Random Forest (RF), Decision Tree (DT), LightGBM (LGBM), Gradient Boosting (GB), Logistics Regression (LR), and XGBoost. ${ }^{14}$ We created our own Python script (see Extended data) using Scikitlearn machine learning library to perform this step. After a careful exploratory data analysis, we dropped Customer ID, Avrg local amt, Avrg std amt, Avrg idd amt, Avrg dialup amt from the predictor variable list since they were weakly correlated to the target variable.

Evaluation of experiment

We performed two evaluations: performance measures ${ }^{15}$ and algorithmic fairness metrics. ${ }^{16}$

\section{Performance measures}

In measuring the classifier's performance, we applied standard measures which are commonly used in most of machine learning classification tasks, including precision, recall and accuracy. We applied F-1 and AUC-ROC scores since accuracy alone is not enough to evaluate the actual performance of the classifiers. We created an own script (see Extended data) using Scikit-learn, a free machine learning software library for Python programming language. The performance of each classification was done as follows:

$$
\text { Accuracy }=\frac{T P+T N}{T P+T N+F P+F N},
$$

where

$$
\begin{aligned}
& \mathrm{TP}=\text { true positive } \\
& \mathrm{TN}=\text { true negative } \\
& \mathrm{FP}=\text { false postive } \\
& \mathrm{FN}=\text { false negative }
\end{aligned}
$$

$$
\text { Precision }=\frac{\text { True positive }}{\text { True positive }+ \text { False positive }}
$$

$$
\text { Recall }=\frac{\text { True positive }}{\text { Ture positive }+ \text { False negative }}
$$$$
\mathrm{F} 1-\text { Score }=2 * \frac{\text { Precision } * \text { Recall }}{\text { Precision }+ \text { Recall }}
$$$$
\mathrm{AUC}-\mathrm{ROC}=\frac{\sum \operatorname{Rank}(+)-|+| *(|+|+1) / 2}{|+|+|-|} \text { where, }
$$

$\Sigma$ Rank (+) is the sum of all positive classified examples

$|+|$ is the number of positive examples in the dataset

$|-|$ is the number of negative examples in the dataset 


\section{Algorithmic fairness metrics}

We emphasized the assessment of whether the classifier is discriminated between women, a protected group, and men, a non-protected group. We applied two well-known fairness definitions in measuring algorithmic fairness, and utilized the popular AI-fairness 360 tool to calculate algorithmic fairness. ${ }^{16}$

Statistical parity $(S P)$ : Also known as an equal acceptance rate. SP is achieved if women have an equal probability to be predicted in the positive, i.e., churn class, as the men. ${ }^{17}$

SP difference measures the difference of a specific outcome between the protected (female group) and non-protected (male group). The smaller the SP difference between the two groups, we can say that the model treats the unprotected group statistically similar to the protected group.

SP is calculated as follows:

$$
\operatorname{Pr}(Y=1 \mid \text { Group }=\text { male })=\operatorname{Pr}(Y=1 \mid \text { Group }=\text { female }), \text { where } Y=\text { predicted decision }
$$

Disparate Impact (DI): Also known as indirect discrimination where no protected variables are directly applied, but biased outcomes are still produced relying on the variables correlated with protected variables. ${ }^{18}$ The standardized threshold in calculation of DI is 0.8 , which means the group whose DI values are under 0.8 are discriminated by the classifier.

The threshold value $80 \%$ is advised by the US Equal Employment Opportunity Commission. ${ }^{19}$ The model could be DI-free when the value is larger than $80 \%$ but it should be lower than $125 \%$ according to. $^{20}$

DI is calculated as follows:

$$
D I=\frac{\operatorname{Pr}(Y=1 \mid \text { Group }=\text { female })}{\operatorname{Pr}(Y=1 \mid \text { Group }=\text { male })} \leq \tau=0.8,
$$

where $Y=$ predicted decision

\section{Results}

The preliminary classification results for the datasets with different data unbalanced rates using four DSTs are shown in Tables 2-4. Table 2 shows the specific results of classification performance gotten when testing on $5 \%$ of unbalanced rate with respect to the chosen classifiers and four DSTs.

Table 3 shows the details results of classification performance obtained when testing on $15 \%$ of unbalanced dataset with respect to the chosen classifiers and four DSTs.

Table 4 shows the details results of classification performance obtained when testing on $30 \%$ of unbalanced dataset with respect to the chosen classifiers and four DSTs.

In our study, we have observed that a variable, is-senior remained unbalanced even after applying the DSTs. The algorithmic fairness scores for each group with different unbalanced rates are described in Tables 5-7. Table 5 shows the comparative results of SP difference and DI scores calculated on 5\% unbalanced dataset and original dataset.

Table 6 displays the comparative results of SP difference and DI scores calculated on $15 \%$ unbalanced dataset and original dataset.

Table 7 describes the comparative results of SP difference and DI scores calculated on $30 \%$ unbalanced dataset and original dataset.

\section{Discussion}

\section{Overview of experimental results}

Recent works of algorithmic fairness research in machine learning applications is broadly organized into three main trends. Some studies emphasize enhancing or proposing better fairness notions and evaluation metrics in line with the 

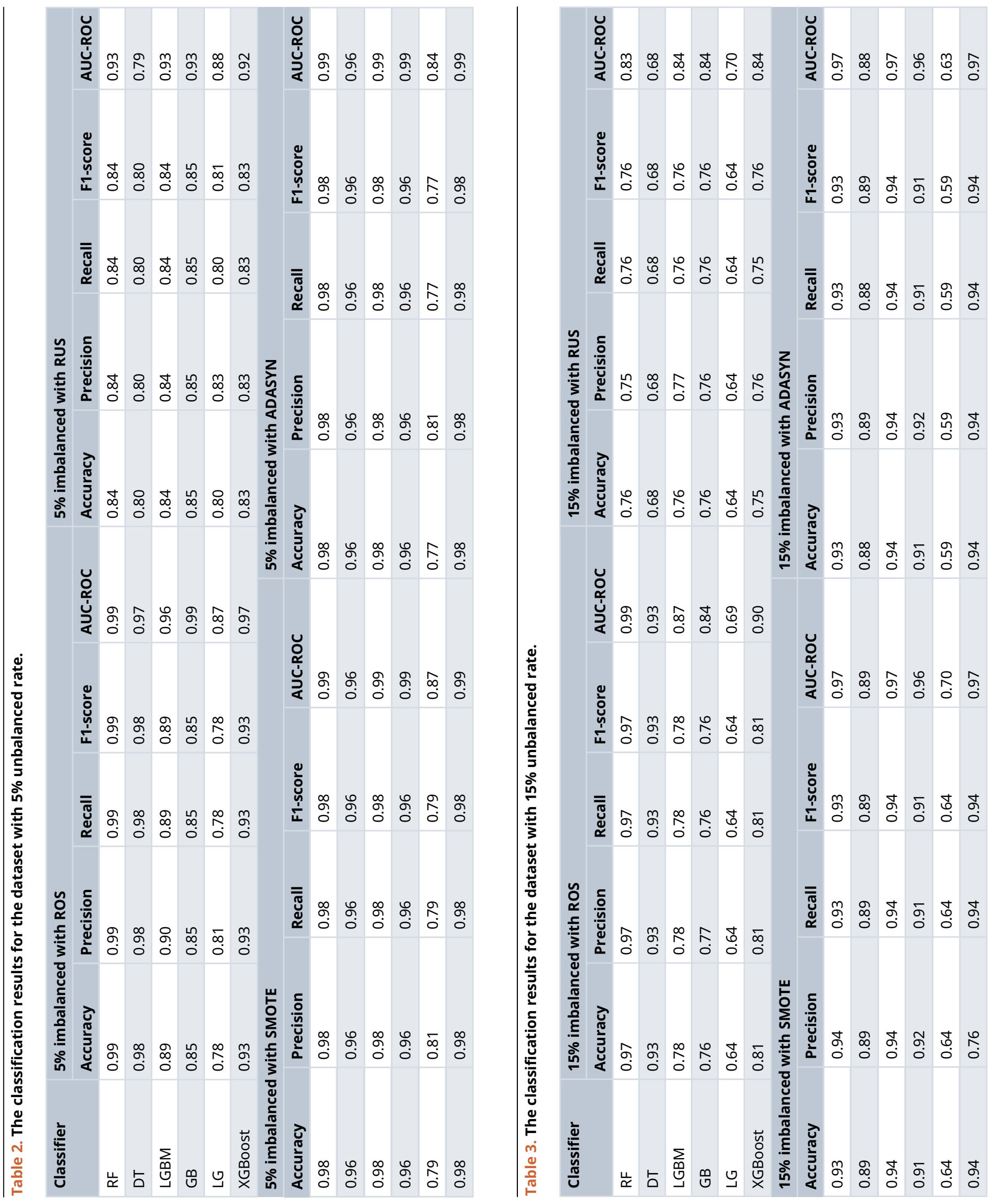


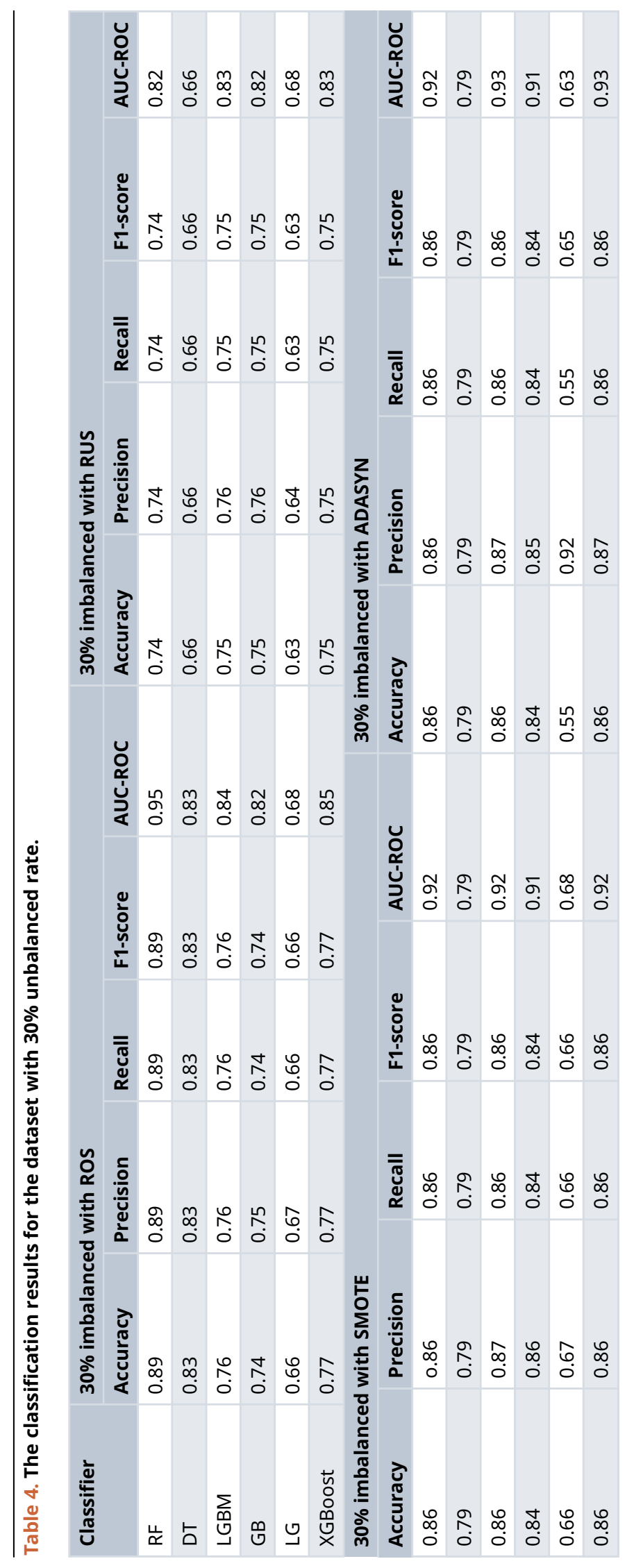


Table 5. The algorithmic fairness measures on $\mathbf{5 \%}$ unbalanced dataset.

\begin{tabular}{|c|c|c|c|c|c|}
\hline $\begin{array}{l}\text { Algorithmic } \\
\text { fairness } \\
\text { metrics }\end{array}$ & $\begin{array}{l}\mathbf{5 \%} \\
\text { original } \\
\text { data }\end{array}$ & $\begin{array}{l}\mathbf{5 \%} \\
\text { imbalanced } \\
\text { with ROS }\end{array}$ & $\begin{array}{l}5 \% \\
\text { imbalanced } \\
\text { with RUS }\end{array}$ & $\begin{array}{l}\mathbf{5 \%} \\
\text { imbalanced } \\
\text { with SMOTE }\end{array}$ & $\begin{array}{l}5 \% \\
\text { imbalanced } \\
\text { with } \\
\text { ADASYN }\end{array}$ \\
\hline \multicolumn{6}{|l|}{ RF } \\
\hline SP Difference & -0.0056 & -0.0703 & -0.0524 & 0.1402 & 0.1401 \\
\hline DI & 0.80 & 0.86 & 0.89 & 1.32 & 1.32 \\
\hline \multicolumn{6}{|l|}{ LightGBM } \\
\hline SP Difference & -0.0067 & -0.0703 & -0.0524 & 0.1402 & 0.1390 \\
\hline DI & 0.79 & 0.86 & 0.89 & 1.32 & 1.32 \\
\hline \multicolumn{6}{|l|}{ GB } \\
\hline SP Difference & -0.0057 & -0.0854 & -0.5456 & 0.1349 & 0.1319 \\
\hline DI & 0.80 & 0.84 & 0.89 & 1.31 & 1.29 \\
\hline \multicolumn{6}{|l|}{ LR } \\
\hline SP Difference & -0.0024 & -0.0789 & -0.0227 & 0.1765 & 0.1591 \\
\hline DI & 0.64 & 0.94 & 0.96 & 1.34 & 1.28 \\
\hline \multicolumn{6}{|l|}{ XGBoost } \\
\hline SP Difference & -0.0069 & -0.0842 & -0.5446 & 0.1387 & 0.1383 \\
\hline DI & 0.78 & 0.85 & 0.89 & 1.32 & 1.32 \\
\hline \multicolumn{6}{|l|}{ DT } \\
\hline SP Difference & -0.0073 & -0.0698 & -0.0397 & 0.1331 & 0.1317 \\
\hline DI & 0.86 & 0.87 & 0.91 & 1.30 & 1.29 \\
\hline
\end{tabular}

Table 6. The algorithmic fairness measures on $15 \%$ unbalanced dataset.

\begin{tabular}{|l|l|l|l|}
\hline $\begin{array}{l}\text { Algorithmic } \\
\text { fairness } \\
\text { metrics }\end{array}$ & $\begin{array}{l}\mathbf{1 5 \%} \\
\text { original } \\
\text { data }\end{array}$ & $\begin{array}{l}\mathbf{1 5 \%} \\
\text { imbalanced } \\
\text { with ROS }\end{array}$ & $\begin{array}{l}\mathbf{1 5 \%} \\
\text { imbalanced } \\
\text { with RUS }\end{array}$ \\
\hline RF & -0.009644 & -0.058223 & -0.049729 \\
\hline SP Difference & 0.871212 & 0.892587 & 0.891628 \\
\hline DI & & & -0.059666 \\
\hline LightGBM & -0.011044 & -0.070341 & 0.872208 \\
\hline SP Difference & 0.856964 & 0.853293 & -0.055826 \\
\hline DI & & & 0.877351 \\
\hline Gradient Boosting & -0.006168 & -0.057517 & \\
\hline SP Difference & 0.913621 & 0.874225 & \\
\hline DI & & & 0.01865 \\
\hline Logistic & & -0.1004 & 1.03868 \\
\hline Regression & -0.007872 & $\mathbf{0 . 7 9 5 8 1}$ & \\
\hline SP Difference & $\mathbf{0 . 7 1 1 7 7 1}$ & & -0.059144 \\
\hline DI & & -0.069346 & 0.874791 \\
\hline XGBoost & -0.011008 & 0.858386 & -0.016812 \\
\hline SP Difference & 0.864076 & & 0.967054 \\
\hline DI & & -0.016556 & \\
\hline Decision Tree & 0.900914 & & \\
\hline SP Difference & & & \\
\hline DI & & & \\
\hline
\end{tabular}


Table 7. The algorithmic fairness measures on $30 \%$ unbalanced dataset.

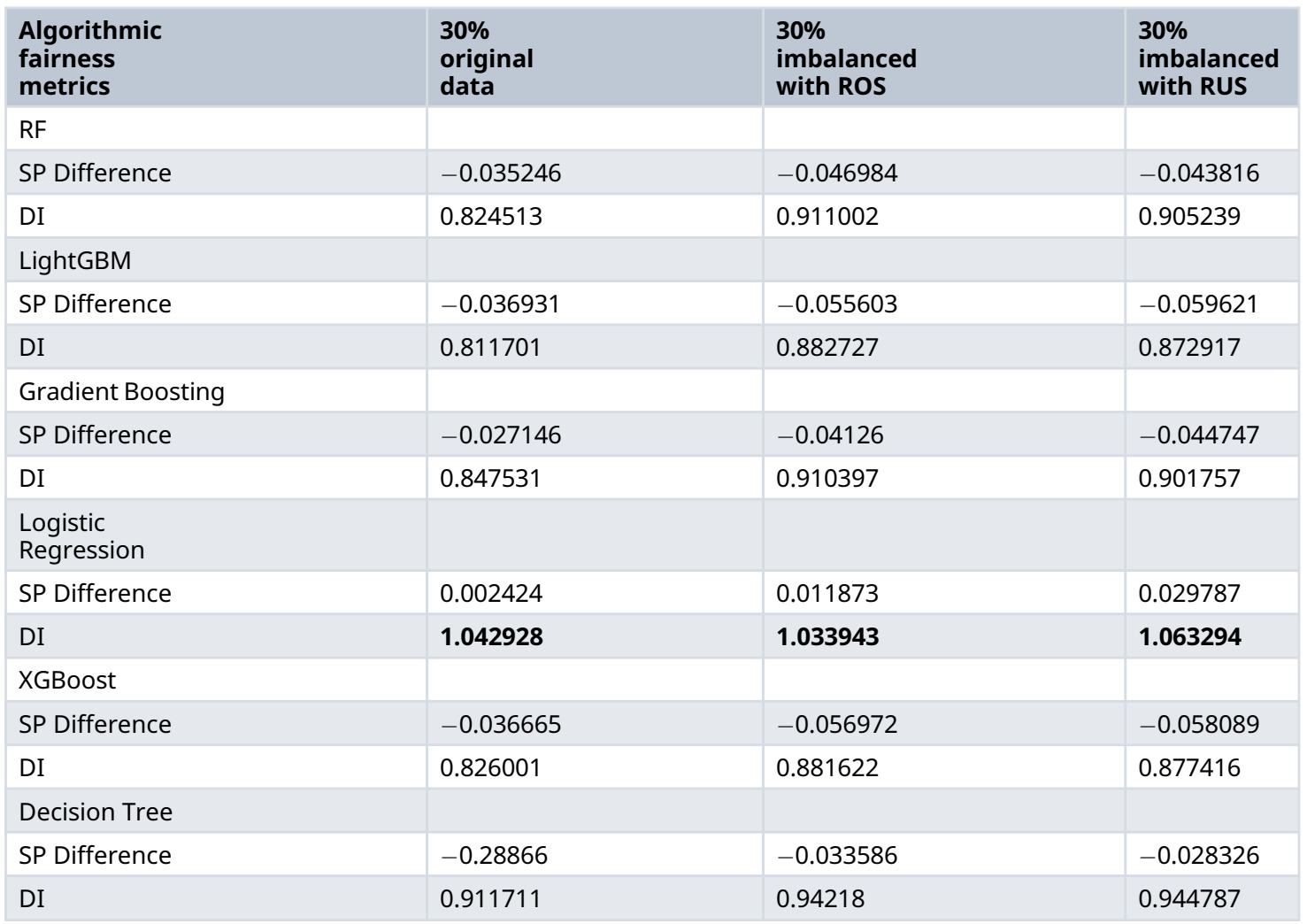

domains concerned, ${ }^{17,21}$ some focus on the ways to mitigate the bias in the classification process (which can further be divided into three main groups: pre-, in-, and post-processing techniques), ${ }^{22-25}$ while the last trend proposes how to maintain the ethical AI standards and policies in practicing machine learning applications in different sectors. ${ }^{26,27}$

Despite some previous empirical studies on the impact of using preprocessing techniques on algorithmic fairness, the findings of previous works could not pinpoint the direct impact of using DSTs on algorithmic fairness. Lourenc and Antunes, ${ }^{28}$ which is the closest work to our research, distinguish the effect of data preparation on algorithmic fairness. However, their work has been tested with two small datasets and provides general results of using random under- and over- DSTs. Importantly, their work fails to be tested on the widely-applied DSTs, SMOTE and ADASYN. In contrast, we apply real-world business data and show how different DSTs influence dissimilar levels of imbalance rate.

In the classification task, RF seems to be the best classifier since it yielded the best results over the other five models, while LR provided the worst scores for almost all metrics. It was observed that RUS worked better for the extremely unbalanced situation compared with $15 \%$ and $30 \%$ imbalanced rates. The best outcomes were found via ROS, SMOTE, and ADASYN in all different unbalanced rates, thus, could be concluded that oversampling techniques seem to provide more promising prediction results over undersampling techniques. This might be because the undersampling technique modifies the data by decreasing the majority of instances, which makes the dataset lack useful information for learning.

For all three unbalanced rates, the original dataset always gave less statistical parity differences (SPD) comparing to sampled datasets created using four DSTs, while datasets with RUS and ROS yield a slightly larger SPD but the statistics showed there is no disparate impact. However, we can hypothetically consider there might still be a bias as both RUS and ROS have their limitations. With RUS, important and essential data could have been removed and the classifier could provide a biased result since there was less information to learn from. On the other hand, with ROS, the prediction performance could also be biased due to the overfitting problem. In this sense, it is suggestible to apply different fairness measures and to compare the fairness scores. For the DI scores, if there is DI less than 0.8 , there is indirect discrimination towards the unprotected group. The mathematical equivalence of DI suggests equalizing the outcomes between protected and unprotected groups. However, in reality, the conditions in the context of interest drive us to allow DI to a specific group up to some percentage. For example, in telecom CCP, the number of female customers could be very less than the 
dataset, since most males usually apply for a network plan representing the whole household. Therefore, we assume considering DI with $80 \%$ rule is reasonable.

In the $5 \%$ unbalanced original dataset, LGBM, LR and XG-Boost imposed with DI values of $0.79,0.64$, and 0.78 respectively. But there is no DI in the other two original datasets for $10 \%$ and $30 \%$. This reveals that more discrimination could occur on a more unbalanced dataset. The analysis on all datasets with SMOTE and ADASYN provides alarming information on the classifier's discrimination on the unprotected group. The $30 \%$ unbalanced dataset yields the worst unfair results since this is the highest SPD between female and males' group with LR as 0.38 and 0.43 , respectively. Overall, among all DSTs, ADASYN, and SMOTE tend to provide more unfair outcomes compared to other DSTs. In contradiction, they both provide a better classification performance in comparison to RUS and ROS. There is not a huge difference among the three different data unbalanced levels. However, in this study, we experimented with the gender attribute as a sensitive variable.

\section{Opportunities and challenges}

Due to the nature of the CCP process and the rarity issue, training datasets have high chances to have compounded bias and suffer from unbalanced problems not only for the target class but also in the other attributes including sensitive variables. We have noticed that one variable remained unbalanced even after applying the DSTs; in such a case, a careful selection of data attributes should be done to avoid selection bias.

As the quality of training data is important, we would suggest enhanced mechanisms of data repairing techniques to prevent bias in the training data. Furthermore, the algorithmic fairness problem is mostly concerns societal discrimination. For example, in the scholarship selection process, if classifiers give more favors to males than females who have the same qualifications as males but are not selected, this will decrease their chances of scholarship. In a profit-centered industry like telecom, one could think there will be no loss for the customers though any group is less or more favored. It is important to consider the impact of biased decisions for the sake of the company's reputation, the importance of equal treatment to customers, and to practice ethical AI policies.

\section{Conclusions}

In this paper, we experimented on three versions of unbalanced real-world telecom datasets to assess the impact of using four types of DSTs on the algorithmic fairness in the CCP process and compared the results with the unsampled original dataset. Classification performance and algorithmic fairness were evaluated with well-known metrics. The outcomes imply that RF provides the best classification results. Using SMOTE and ADASYN yields larger SPD between male and female groups as well as a disparate impact on the female over the male group. Previous work emphasizes the use of this method in choosing a scholarship candidate, releasing prisoners on parole, and choosing a credit candidate. Since machine learning applications would be applied to almost every sector in the near future, the practice of using fairer or unbiased systems is essential. Our study highlights the importance of paying attention to algorithmic fairness in the machine-driven decision-making process of the profit-centered and customer-oriented sectors on which very little research work has been done. Particularly, our finding highlights the fact that a careful choice of DSTs must be done to achieve unbiased prediction results. In future work, we would like to test the same procedure on a larger dataset and would like to measure more algorithmic fairness metrics to investigate the best suitable algorithmic measures for the CCP task. Moreover, we would like to test more sensitive variables rather than just gender.

\section{Data availability}

Underlying data

The real-world telecom dataset was obtained from the Business Intelligence and Analytics department of Telekom Malaysia Bhd. The authors were required to go through a strict approval process following established data governance framework. Interested readers/reviewers may contact the Business Intelligence and Analytics department to request the data (technicalsuport@tm.com.my). The decision as to whether or not to grant access to the data is at the discretion of Telekom Malaysia Bhd.

As most telco companies own similar customer data, other customer churn datasets that are representative of the data being used in this research can be found as follows:

1. https://www.ibm.com/docs/en/cognos-analytics/11.1.0?topic=samples-telco-customer-churn.

2. https://datasetsearch.research.google.com/search?query=Telco\%20Customer\%20Churn\%20dataset $\% 20$ site $\%$ 3Akaggle.com\&docid=L2cvMTFsbDF0dzJ5NA\%3D\%3D. 
Extended data

Analysis code available from: https://github.com/mawmaw/fairness_churn.

Archived analysis code as at time of publication: https://doi.org/10.5281/zenodo.5516218. ${ }^{29}$

License: MIT License.

1. Eria K, Marikannan BP: Systematic Review of Customer Churn Prediction in the Telecom Sector. 2018; vol. 2(no. 1).

2. Amin S, Anwar, et al.: Comparing Oversampling Techniques to Handle the Class Imbalance Problem: A Customer Churn Prediction Case Study. IEEE Access. 2016; 4(MI): 7940-7957. Publisher Full Text

3. Gui C: Analysis of imbalanced data set problem: The case of churn prediction for telecommunication. 2017; vol. 6(no. 2): pp. 93-99.

4. Dieterich W, Mendoza C, Brennan T: COMPAS Risk Scales: Demonstrating Accuracy Equity and Predictive Parity. 2016.

5. Ntoutsi E, et al.: Bias in Data-driven AI Systems -- An Introductory Survey. 2020; pp. 1-19.

6. Kamiran F, Žliobaitè I: Explainable and non-explainable discrimination in classification. Stud. Appl. Philos. Epistemol. Ration. Ethics. 2013; 3(January 2012): 155-170. Publisher Full Text

7. Barocas S, Selbst AD: Big Data's Disparate Impact. 2016; vol. 671: pp. 671-732.

8. Ballings M, Van Den Poel D, Verhagen E: Improving customer churn prediction by data augmentation using pictorial stimulus-choice data. Adv. Intell. Syst. Comput. 2012; 171(1): 217-226.

Publisher Full Text

9. Columelli L, Nunez-Del-Prado M, Zarate-Gamarra L: Measuring churner influence on pre-paid subscribers using fuzzy logic. Proc. 2016 42nd Lat. Am. Comput. Conf. CLEI 2016. 2017.

10. Ahmed $\mathrm{U}, \mathrm{Khan} \mathrm{A}$, Khan SH, et al.: Transfer Learning and Meta Classification Based Deep Churn Prediction System for Telecom Industry. 2019; pp. 1-9.

11. Mohammed R, Rawashdeh J, Abdullah M: Machine Learning with Oversampling and Undersampling Techniques: Overview Study and Experimental Results. 2020 11th Int. Conf. Inf. Commun. Syst. ICICS 2020. 2020; 243-248.

12. Chawla NV, Bowyer KW, Hall LO: SMOTE: Synthetic Minority Oversampling Technique. 2002; 16: 321-357.

13. Gosain A, Sardana S: Handling class imbalance problem using oversampling techniques: A review. 2017 Int. Conf. Adv. Comput. Commun. Informatics, ICACCI 2017. 2017; 2017-January: 79-85.
14. Kotsiantis SB: Supervised Machine Leanring: A Review of Classification Techniques. Informatica. 2007; 31(2007): 249-268.

15. Kotu V, Deshpande B: Model Evaluation. Predict. Anal. Data Min. 2015; 257-273. Publisher Full Text

16. Bellamy RKE, et al.: AI Fairness 360: An Extensible Toolkit for Detecting, Understanding, and Mitigating Unwanted Algorithmic Bias. 2018.

17. Dwork $C$, Hardt $M$, Pitassi $T$, et al: Fairness through awareness. ITCS 2012 - Innov. Theor. Comput. Sci. Conf. 2012; 214-226.

18. Calmon FP, Wei D, Ramamurthy KN, et al.: Optimized Data PreProcessing for Discrimination Prevention. 2017; 1-18.

19. Feldman $M$, Friedler SA, Moeller J, et al.: Certifying and removing disparate impact. Proc. ACM SIGKDD Int. Conf. Knowl. Discov. Data Min. 2015; 2015-Augus: 259-268.

20. Zafar MB, Valera I, Rodriguez MG, et al.: Fairness Constraints: Mechanisms for Fair Classification. 2017; 54.

21. Hardt $M$, Price $E$, Srebro N: Equality of opportunity in supervised learning. Adv. Neural Inf. Proces. Syst. 2016; 3323-3331.

22. Zemel R, Wu Y, Swersky $\mathrm{K}$, et al.: Learning fair representations. 30th Int. Conf. Mach. Learn. ICML 2013. 2013; 28(PART 2): 1362-1370.

23. Calmon FP, Wei $D$, Vinzamuri B, et al.: Optimized pre-processing for discrimination prevention. Adv. Neural Inf. Proces. Syst. 2017; 2017. Decem(Nips): 3993-4002.

24. Zhang $B$ H, Lemoine $B$, Mitchell $M$ : Mitigating Unwanted Biases with Adversarial Learning. AIES 2018- Proc. 2018 AAAI/ACM Conf. AI, Ethics, Soc. 2018; 335-340.

25. Pleiss G, Raghavan M, Wu F, et al.: On fairness and calibration. $A d v$. Neural Inf. Proces. Syst. 2017; 2017-Decem(Nips): 5681-5690.

26. Gursoy ME, Tamersoy A, Truex S, et al.: Secure and Utility-Aware Data Collection with Condensed Local Differential Privacy. IEEE Trans. Dependable Secur. Comput. 2019; X: 1-1.

27. Hube C, Fetahu B, Gadiraju U: Understanding and mitigating worker biases in the crowdsourced collection of subjective judgments. Conf. Hum. Factors Comput. Syst. - Proc. 2019.

28. Lourenc N, Antunes N: The Impact of Data Preparation on the Fairness of Software Systems.

29. mawmaw: mawmaw/fairness_churn: Initial (v1.0). Zenodo. 2021. Publisher Full Text 


\section{Open Peer Review}

\section{Current Peer Review Status: ? ?}

\section{Version 1}

Reviewer Report 24 February 2022

https://doi.org/10.5256/f1000research.76542.r120732

(C) 2022 P P. This is an open access peer review report distributed under the terms of the Creative Commons Attribution License, which permits unrestricted use, distribution, and reproduction in any medium, provided the original work is properly cited.

\section{Prabu P}

Department of Computer Science, CHRIST University, Bengaluru, Karnataka, 560029, India

This work mainly focus on data imbalance problems in customer churn prediction. The author has to incorporate the following suggestion in his/her article in-order to improve the quality of the work

1. The author need to clearly defined performance metrics for comparing random forest classifier with other classifiers.

2. More experimental studies required for unequal balancing datasets and author expected to try the experiment with large datasets.

3. Author need to identify some proper technique to manage outliers in the datasets instead of removing manually.

4. Results and Algorithms fairness need to be explain properly.

5. Introduction section are too short and the section is not continuous. Correct it. The introduction section is not clearly explaining the basics and also lack of some details of about "issues in existing work, scope of work, goal of work and organization of work" can be included.

6. No significant limitations are discussed. A number of limitations and learning points are also be considered after the conclusion.

7. Author could take more recent research papers for the literature review and most of the literature review papers are not suitable to the proposed work. Author is expected to include few existing recommender system suggested for e-commerce platform. So I would suggest the author should identify few more recent papers based on his proposed research work and also include the objective and limitations of each work in table format.

Is the work clearly and accurately presented and does it cite the current literature? 
Yes

Is the study design appropriate and is the work technically sound?

Yes

Are sufficient details of methods and analysis provided to allow replication by others? Yes

If applicable, is the statistical analysis and its interpretation appropriate? Partly

Are all the source data underlying the results available to ensure full reproducibility? Partly

Are the conclusions drawn adequately supported by the results?

Yes

Competing Interests: No competing interests were disclosed.

Reviewer Expertise: Machine Learning, Cloud Computing ad Mathematical Modeling

I confirm that I have read this submission and believe that I have an appropriate level of expertise to confirm that it is of an acceptable scientific standard, however I have significant reservations, as outlined above.

\section{Author Response 06 Jun 2022}

Haw Su Cheng ., Multimedia University, Cyberjaya, Malaysia

1. AUC-ROC score was applied to compare the performance of the respective classifiers and this information is added for clarification in the discussion section/ performance measure section

2. Due to the dataset availability on propriety company, the focus on this paper is on 100,000 records only. Testing with large dataset is put as future work. In future work, we would like to test the same procedure on a larger dataset and would like to measure more algorithmic fairness metrics to investigate the best suitable algorithmic measures for the CCP task.

3. Since there were not a lot of outliers in the original dataset, they were manually removed and we added this fact in the data pre-processing section.

4. In our study, we do not go further process of mitigating algorithmic unfairness yet. If so, we will need to apply and discuss. But here, we only evaluate and compare the bias in the training dataset before and after applying the data sampling techniques.

5. Introduction section is updated with related works, scope of work and organization 
work. Since my background is the combination of three different topics, algorithmic fairness, customer churn prediction and data sampling techniques, Due to the page limitation, could not put a lot of materials in the introduction section but most important and relevant background study was provided in the introduction section in a compact way. Goal of work is provided in the last paragraph of the introduction section, "The main goal of this study is to explore and identify the impact of using DSTs on training data on algorithmic fairness in the CCP process."

6. We updated with the limitations in the conclusion section as per suggestion.

7. Although there is very limited work which are related to my work, we organize some of the articles of fairness in recommender system in the table format as per your suggestion

Competing Interests: No competing interests were disclosed.

Reviewer Report 11 January 2022

https://doi.org/10.5256/f1000research.76542.r96164

(C) 2022 Loo C. This is an open access peer review report distributed under the terms of the Creative Commons Attribution License, which permits unrestricted use, distribution, and reproduction in any medium, provided the original work is properly cited.

\section{Chu Kiong Loo}

Faculty of Computer Science and Information Technology, University of Malaya, Kuala Lumpur, Malaysia

This paper addresses an important issue of algorithmic fairness, i.e. to investigate whether the results pose any discrimination between male and female groups and compare the results before and after using DSTs.

I suggest to add some discussions on confusion matrix, learning curve and the improvement of fairness index before and after implementing the DSTs.

Is the work clearly and accurately presented and does it cite the current literature? Yes

Is the study design appropriate and is the work technically sound? Yes

Are sufficient details of methods and analysis provided to allow replication by others? Yes 
If applicable, is the statistical analysis and its interpretation appropriate?

Yes

Are all the source data underlying the results available to ensure full reproducibility?

Yes

Are the conclusions drawn adequately supported by the results?

Yes

Competing Interests: No competing interests were disclosed.

Reviewer Expertise: Machine learning

I confirm that I have read this submission and believe that I have an appropriate level of expertise to confirm that it is of an acceptable scientific standard, however I have significant reservations, as outlined above.

Author Response 06 Jun 2022

Haw Su Cheng ., Multimedia University, Cyberjaya, Malaysia

Learning curves for Random Forest before and after applying DSTs (for three versions of datasets) are provided. We discussed briefly in the discussion section as well.

Competing Interests: No competing interests were disclosed.

The benefits of publishing with F1000Research:

- Your article is published within days, with no editorial bias

- You can publish traditional articles, null/negative results, case reports, data notes and more

- The peer review process is transparent and collaborative

- Your article is indexed in PubMed after passing peer review

- Dedicated customer support at every stage

For pre-submission enquiries, contact research@f1000.com

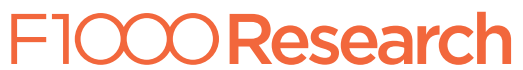

